\title{
A Framework to Leverage Domain Expertise to Support Novice Users in the Visual Exploration of Home Area Networks
}

\author{
Yuqian Song \\ FAME \& KDEG \\ School of Computer Science and \\ Statistics \\ Trinity College Dublin \\ Dublin, Ireland \\ yuqians@scss.tcd.ie
}

\author{
John Keeney \\ Network Management Lab \\ LM Ericsson \\ Athlone \\ Co. WestMeath \\ Ireland \\ John.Keeney@ericsson.com
}

\author{
Owen Conlan \\ FAME \& KDEG \\ School of Computer Science and \\ Statistics \\ Trinity College Dublin \\ Dublin, Ireland \\ owen.conlan@scss.tcd.ie
}

\begin{abstract}
Advances in modern technologies have afforded endusers increased convenience in performing everyday activities. However, even seemingly trivial issues can cause great annoyance for the ordinary user who lacks domain expertise of the often complex systems that underpin these advances. A key challenge lies in assisting non-expert users to express their requirements of an obscure and complex system. This research proposes a semantic approach by using domain expert knowledge to enable real time semantic up-lift in supporting novice end-users to understand and control the complex dynamic systems they must manage. This presents a significant opportunity to increase user satisfaction and reduce associated support costs. This semantic approach has been designed and implemented in an early prototype of our Home Area Network Monitoring System (HANMS). This paper presents a detailed description of the current state of the research, an initial evaluation, and future work.
\end{abstract}

Keywords-component; formatting; style; styling; insert (key words)

\section{INTRODUCTION}

Home Area Network (HAN) technologies are becoming more and more pervasive and complex. Such HANs require device management skills and knowledge to optimize how well the home network is used. Inexperienced users are continuously challenged and perplexed with even simple HAN management and maintenance tasks [10]. For example, an important VoIP call suddenly suffers degradation of wireless network connection quality, perhaps caused by a neighbor initiating some WiFi activity on the same channel - causing interference, or a signal weakens when the device antenna is obstructed, or another user may upload a large file which causes network congestion. Such events may be unremarkable but will impact on the end-user's perception of the quality of the service supported by the wireless network connection. Additional cost for both the network provider and HAN user will be incurred to diagnose and resolve such issues. Moreover, the solution of such events may involve a series of optimizations and controls of HAN resources that most home users will not have the technical expertise to implement.
This research addresses the challenge of how domain expert knowledge may be applied to enable the real time semantic uplift of meaningful information from raw data to support novice end-users to understand and control complex dynamic systems. To address this challenge, this research proposes a framework to bridge the semantic gap between the hard-learned knowledge of domain experts and the cognitive competencies of normal users in a HAN context.

This framework aims to achieve the following objectives:

- Capture and model the insights and knowledge of the domain expert for the HAN monitoring;

- Up-lift meaningful information from raw data (e.g. wireless network log data in a HAN scenario) through leveraging captured expert knowledge in a real-time dynamic process;

- $\quad$ Adopt knowledge reasoning to facilitate the diagnosis and analysis of anomalies;

- Decompose high-level control and management goals from non-expert users into low-level management actions or re-configurations;

- Present an organized, correlated and annotated view of the up-lifted information to enable non-expert users to understand potentially obscure details of complex dynamic systems.

This paper is organized as follows: Related research already addressing some of these objectives will be briefly reviewed in the Related Work section. The System Design section introduces a framework for the proposed approach. The Implementation and Evaluation section presents an early prototype of the approach applied to HAN management, followed by preliminary evaluation results. Future work and conclusions are described in the final section.

\section{RELATED WORK}

Up to 2009 , over $99 \%$ of UK households were connected to a broadband enabled exchange, while the global network monitoring and management market is over $\$ 1.9$ billion [11]. 
In this rapidly evolving area, a significant challenge lies in how to extract meaningful information from the vast number of dynamic network log datasets. Driven by Semantic Web technologies, Semantic Annotation can be applied to transform and enrich the data and information with formal semantic meanings. Handschuh and Staab [1] introduce a broad range of technologies and methods for the explicit construction of semantic annotations, including approaches for data extraction, collaboration and translation into Semantic Web metadata. SARA [2] can be used to gather heterogeneous data from different resources and endow the raw data with semantic attributes through logic/rules specified by domain experts. Adopting such an approach to enhance and assist the network management process is challenging however due to the highly dynamic nature of network monitoring data.

The continuous evolution and heterogeneity of HAN technologies coupled with varying user requirements poses a significant challenge. One novel and effective strategy to overcome this complexity is to support network management by involving ontology-based modeling and reasoning; e.g., Hoag [3] presents an approach to apply semantic reasoning techniques for network management and resource allocation to avoid overbuilding and improve quality. FOCALE [4] is a semantically rich architecture for orchestrating the behavior of heterogeneous and distributed computing resources. However, further research is still required in this area to leverage the correlated knowledge to support high-level management goals via low-level network management actions more effectively in the management process.

Visualization techniques can effectively present complex data by capitalizing on human perception capabilities, and a novel interactive visualization system [5] was proposed as an approach to monitor the data collected from a home router and control bandwidth usage for family users. Another interactive network management system, Eden [6], delivers a simple conceptual model to help users understand key aspects of the network by eliminating the barrier and cost of the network technical minutia. Our research is also based on [7] to express a semantically enriched visualization approach for non-expert users to monitor networks and services.

\section{FrameWORK DESIGN}

According to objectives above, this section describes a framework (Figure 1) to capture and model domain-expert knowledge to enable the real time semantic up-lift of meaningful monitoring information from raw monitoring data to support novice end-users in understanding and controlling their HAN. This framework is established with two main components: Domain Expert Authoring Tool (DEAT) and Home Area Network Monitoring System (HANMS).

\section{A. Domain Expert Authoring Tool (DEAT)}

This tool aims to capture the insights of domain experts and extract the domain knowledge model via an interactive visual interface. Through the knowledge extraction process, captured domain expert insights, including schemas, rules, and procedures, are associated with domain ontologies and modeled in a domain expert knowledge model, which is partly

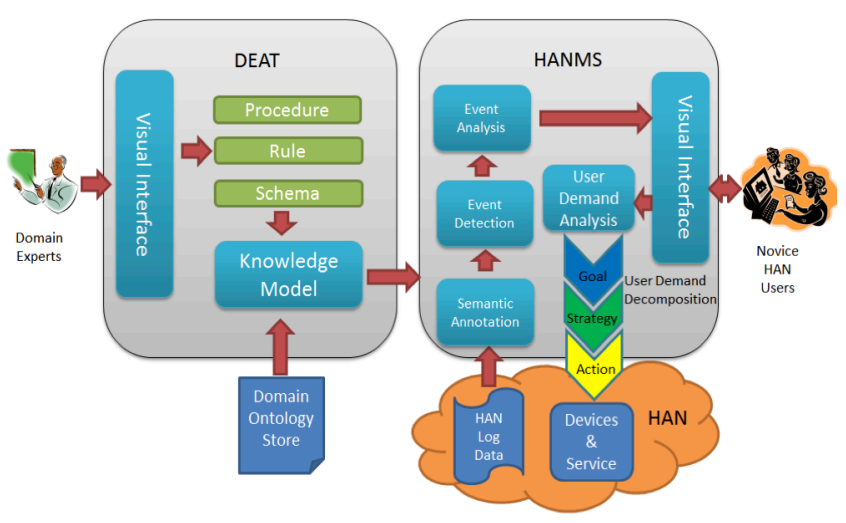

Figure 1: Framework Design

exposed in Figure 2. In this model, there are four main ontology classes: Condition, Event, Reason, and Solution. The Condition class indicates the condition which could be used to trigger an event. Based on condition rules, one condition could contain one or several semantic attributes, which are pieces of semantic encodings captured from domain experts. For example, the condition to trigger the event Interference is composed by two semantic attributes: AntennaNoise_Bad and SignalStrength_Good. The Event class is used to describe the network problem or network status. Events could also be conditions to trigger other events. The relationship between Condition and Event could be described as an if-then rule, which could be automatically reasoned over by the ontology reasoner. Event instances are also associated with the HAN component: device, service, gateway or the HAN itself.

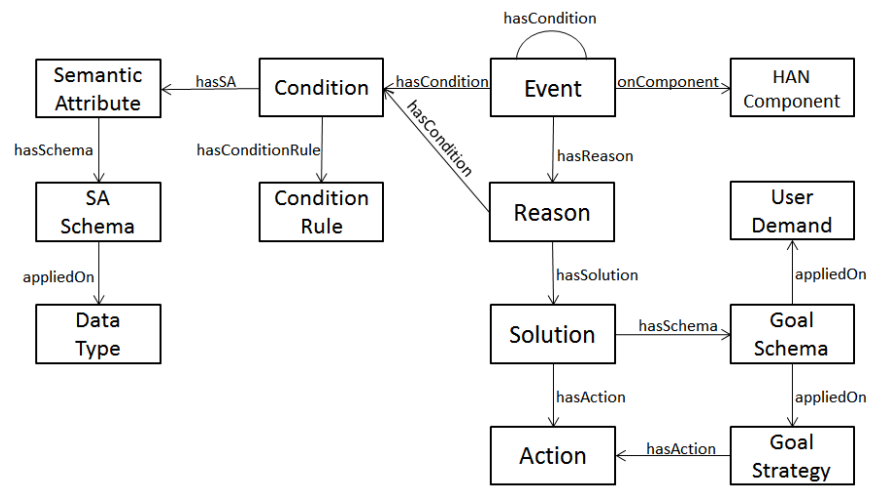

Figure 2: Domain Expert Knowledge Model

The Reason class indicates the expert-defined reasons which may cause an event of a given type. Reason also has conditions which could be considered as an if-then rule for the ontology reasoner to analyze whether the event is actually caused by this reason. For example, OutOfRange could be the reason for events of type SignalLost.

The Solution class describes expert-defined solutions which could be applied against one particular reason of the event. A solution can then be enacted if the suggested candidate solution is in accordance with the goals of the user. Such goals are defined a Goal Schemas, so the solution with a corresponding Goal Strategy can be executed by way of the Solution's corresponding Actions. 




Figure 3: HANMS Prototype Screenshot

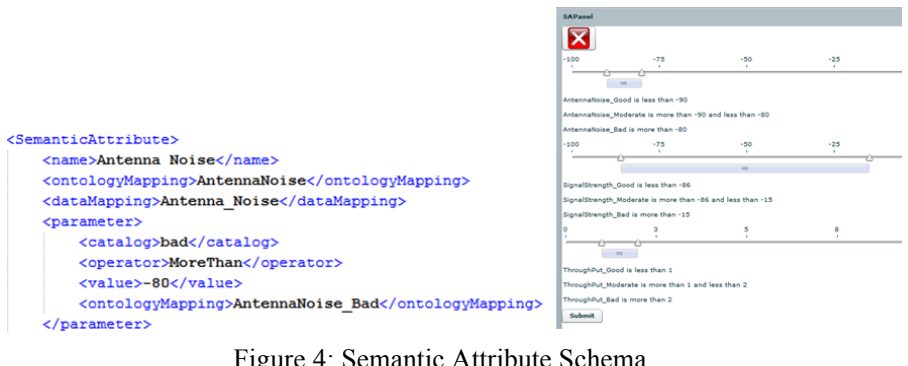

Figure 4: Semantic Attribute Schema

As mentioned in the domain knowledge model, the Semantic Attribute Schema, Goal Schema, and Goal Strategy are all schemas captured from domain experts. These linked named schemas are XML-based 'capsules' of insight from domain experts. As shown in Figure 4, the left-hand side is a piece of schema to bridge the gap between the data metric "Antenna_Noise" from the raw $\log$ data and the semantic attribute AntennaNoise_Bad, where restrictions and thresholds can be adjusted by domain experts via the visual widget on the right. These condition rules in the knowledge model, which can be cascaded, are currently encoded in simple if-then SWRL [8] rules. These can be loaded, parsed and evaluated by the semantic reasoner. For instance, if AntennaNoise_Bad and SignalStrength_Good, then Interference. The Procedure is used to encode the sequence of information uplift and the HAN management process. For the HAN management procedure, the process sequence is Detection, Analysis, Strategy, and Action.

\section{B. Home Area Network Monitoring System (HANMS)}

HANMS [9] presents an approach driven by the domain knowledge model (derived from $D E A T$ ) to enable the real time semantic up-lift of meaningful monitoring information from raw data to support novice end-users in understanding and controlling Home Area Network. This approach is mainly composed by two processes:

\section{1) Information Up-lifting Process}

The information up-lift process is a multi-layer process. In a given time interval, wireless log data of all monitored devices and services in the HAN is collected and aggregated. According to the expert-defined semantic attribute schema, the raw data is annotated with corresponding semantic attributes. There are three layers for this semantic annotation $\operatorname{process}(P)$ :
The first layer is the real-time semantic attribute annotation, which could be considered as a sequence of semantic meaning points $(S)$ annotated for a real-time data stream, i.e. $P=\left\{S_{1} \ldots, S_{m}\right\}$, where $S_{i}=(s, t)$ is a pair with the semantic meaning (s) at timestamp $t$. The second layer is the high-level semantic attribute annotation. The high-level semantic attribute with the corresponding semantic meaning $(s)$ is determined according to other semantic attributes $\left(s_{1 . .,}, s_{n}\right)$, i.e. $P=\left\{S_{1} \ldots, S_{m}\right\}$, where $S_{i}=\left(S,\left\{S_{1 . .,} S_{n}\right\}\right)$. The third layer is the behavior semantic attribute annotation, which is based on behavior events in the raw data, i.e. $P=\left\{S_{1} \ldots, S_{m}\right\}$, where $S_{i}=\left(b, t_{1}, t_{2}\right)$ is a triple with the behavior (b) happened in a period $\left(t_{1}, t_{2}\right)$, and e.g. "Play" is a behavior for an IPTV service. When the IPTV service is playing, the semantic attribute playing is dynamically annotated into the log data flow.

In this multi-layer annotation process, semantically meaningful information is extracted from raw data. According to annotated semantic attributes, all related events in the domain knowledge model are checked one by one in an event diagnosis loop, in which the information is iteratively annotated with events from low-level to high-level. For example, the semantic attribute could be considered as a lowlevel annotation, and if there is another event whose condition is based on this initial annotation we can refer higher-level events, and so events are annotated level by level. All annotated events are kept in an event pool. In the up-lifting process, the event pool constantly checks the semantic annotation loop until there are no more new events (and no rules to fire) and at that time, the up-lifting of the data in this time interval is finished. The events in the event pool are then maintained for use in the management process.

2) HAN management Process

Driven by the domain knowledge model, the event analysis engine reviews all the events in the event pool to evaluate the health and status of all devices and services in the HAN and detect existing and potential problems. The analysis result is represented in the visual user interface (figure 3), which allows novice HAN users to explore what is happening, what will happen, what caused the problem, and the available solutions. 
With this HAN monitoring interface, novice users can then also manage and control the HAN by expressing their demands (based on template User Demand schemas). The user interface could capture high level requirements of novice users which are then transformed into management strategies to indicate appropriate solutions for devices/services in the HAN. Moreover, as described above such high-level User Demands are associated with expert-defined Goal Strategies, which contain low-level reconfiguration actions which can be directly applied to the device/service. In an implemented case study (figure 3), there are three devices in a HAN: VoIP on an iPad, IPTV on an Xbox, and a download service on a laptop. A novice HAN user expresses their demand to enhance the important VoIP call from low quality to good quality using the HANMS interface. When network congestion occurs, based on the User Demand, the corresponding strategy is to limit the throughput of other services in the HAN. This strategy is then decomposed into throughput limitation actions applied to both IPTV service on the Xbox and download service on the laptop. The management process and the re-configuration result are also presented on the visual interface.

\section{IMPLEMENTATION AND EVALUATION}

\section{A. Implementation}

As shown in Figure 3, a prototype of Home Area Network Monitoring System (HANMS) has been developed and deployed from a local server. Raw wireless log data was collected from a physical wireless monitoring test-bed [9] and loaded into a simulation engine for demo and test purposes. HANMS is fed by the simulation engine to generate several wireless device log data streams every second seeded by the data collected from real wireless devices. The expert defined schemas are stored in an XML database, which can be adjusted via a simple widget (right hand side of figure 3 ). The serverside HANMS logic, which imports schemas and domain ontologies, was developed in J2EE and deployed on a Tomcat server in the local network and the client-side visual components are implemented in Flex.

\section{B. Evaluation}

The current HANMS prototype has been initially evaluated under three different tasks with two target groups: novice HAN users (19 volunteers) and domain experts (5 experts) [9]. Novice HAN users have no or limited network technology background. In these tasks, participants were required to use HANMS prototype to detect three different HAN problems by themselves. They then completed a SUS-based feedback questionnaire on the functionality, effectiveness, efficiency, usability, user-interface, and limitations of HANMS. Most participants agree that HANMS is usable by a non-expert user. More than $70 \%$ of participants believe HANMS could detect and analyze common network problems effectively. As a result of the test tasks, 50 out of 57 HANMS observations were correct, which indicates that even in its current prototype state HANMS is suitable for different tasks in different scenarios. Moreover, nearly all participants agree that HANMS is more efficient than traditional network monitoring tools. Identified limitations will be addressed in future development iterations.

\section{FURTHER WORK AND CONCLUSION}

This paper has introduced a framework to capture, model and leverage domain expert knowledge to enable the real time semantic up-lift of meaningful information from raw data in order to support novice end-users in exploring their HAN by themselves. This paper also highlights the knowledge extraction process, information up-lifting process and ontology-driven network management process of this framework. The current prototype is still under active development. The evaluation results show promise and HANMS has the potential to support rich features. Future releases of the prototype will further demonstrate how semantic web technologies can improve traditional network management applications in different application scenarios.

\section{ACKNOWLEDGMENT}

This work is partly funded by Science Foundation Ireland via grant 08/SRC/I1403 - Federated, Autonomic Management of End-to-End Communications Services (FAME).

\section{REFERENCES}

[1] S. Handschuh, S.Staab, "Annotation for the Semantic Web," IOS Press 2003.

[2] C. Hampson, and O. Conlan, "Supporting Personalized Information Exploration through Subjective Expert-created Semantic Attributes," 2009 IEEE International Conference on Semantic Computing, IEEE, 2009, p. 384-389.

[3] J.C. Hoag and F.A. Hayes-Roth, "Semantic Reasoning for Adaptive Management of Telecommunications Networks," IEEE International Conference on Systems, Man and Cybernetics, SMC'06, Taipei, Taiwan 2006, p. 127-131.

[4] J. Strassner, N. Agoulmine, and E. Lehtihet, "Focale: A novel autonomic networking architecture,” Multimedia Systems, 2006, pp. 48-60.

[5] M. Chetty, R. Banks, R. Harper, T. Regan, A. Sellen, C. Gkantsidis, T. Karagiannis, and P. Key, "Who's hogging the bandwidth: the consequences of revealing the invisible in the home," Proceedings of the 28th international conference on Human factors in computing systems, ACM, 2010, p. 659-668.

[6] J. Yang, W.K. Edwards, and D. Haslem, "Eden: supporting home network management through interactive visual tools," Proceedings of the 23nd annual ACM symposium on User interface software and technology, ACM, 2010, p. 109-118.

[7] O. Conlan, J. Keeney, et al., "Towards Non-expert Users Monitoring Networks and Services through Semantically Enhanced Visualizations," Proceedings of the 6th International Conference on Network and Service Management (CNSM 2010), Niagara Falls, Canada , 2010.

[8] "SWRL: A Semantic Web Rule Language Combining OWL and RuleML", http://www.w3.org/Submission/SWRL/

[9] Y. Song, J. Keeney, P. Perry, A. Hava, O. Conlan, “An Ontology-driven Approach to support Wireless Network Monitoring for Home Area Networks," Proceedings of the 7th International Conference on Network and Service Management (CNSM 2011), Paris, France, 2011.

[10] Yankee Group, "IVPN Service Backbones: The Operations Cost Angle", Data Communications Report Vol. 13, No.19, Yankee Group, December 1998.

[11] D. Emma, "Broadband Access", Key Issues for the New Parliament 2010, UK Parliament. http://www.parliament.uk/documents/commons/lib/research/key\%20issu es/Key $\% 20$ Issues $\% 20$ Broadband $\% 20$ access.pdf 